JAHRBUCH DER JEAN-PAUL-GESELLSCHAFT

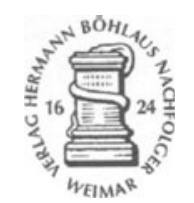




\title{
JAHRBUCH DER \\ JEAN-PAUL-GESELLSCHAFT
}

\author{
IM AUFTRAG \\ DER JEAN-PAUL-GESELLSCHAFT, SITZ BAYREUTH \\ HERAUSGEGEBEN VON \\ HELMUT PFOTENHAUER
}

35./36. JAHRGANG 
Das Jahrbuch erscheint als Jahresgabe an die Mitglieder der Jean-Paul-Gesellschaft für 2000/2001. Überweisung des Jahresbeitrags - für Ordentliche und Korporative Mitglieder DM 40,- für Studenten DM 25,- jeweils zum Jahresanfang auf das Konto der Gesellschaft.

Richtlinien zur Erstellung der Manuskripte sind zu erfragen bei der Redaktion des Jahrbuches:

Redaktion des Jean-Paul-Jahrbuchs

Universität Würzburg

Institut für Deutsche Philologie

Neuere Abteilung

Am Hubland

97074 Würzburg

Redaktion dieses Bandes: Dr. Michael Will

Die Deutsche Bibliothek - CIP-Einheitsaufnahme

Jean-Paul-Gesellschaft:

Jahrbuch ... der Jean-Paul-Gesellschaft. -

Weimar : Verlag Hermann Böhlaus Nachfolger Weimar

Erscheint jährlich. - Früher im Verl. Mühl, Bayreuth. -

Aufnahme nach Jg. 34. 1999

ISSN 0075-3580

Jg. 34. 1999

ISBN 978-3-7400-1158-1

ISBN 978-3-476-02794-8 (eBook)

DOI 10.1007/978-3-476-02794-8

ISSN 0075-3580

Alle Rechte vorbehalten. Ohne schriftliche Genehmigung des Verlages ist es nicht gestattet, das Werk unter Verwendung mechanischer, elektronischer und anderer Systeme in irgendeiner Weise zu verarbeiten und zu verbreiten. Insbesondere vorbehalten sind die Rechte der Vervielfältigung - auch von Teilen des Werkes - auf photomechanischem oder ähnlichem Wege, der tontechnischen Wiedergabe, des Vortrags, der Funk- und Fernsehsendung, der Speicherung in Datenverarbeitungsanlagen, der Übersetzung und der literarischen oder anderweitigen Bearbeitung.

C) 2001 Springer-Verlag GmbH Deutschland Ursprünglich erschienen bei Verlag Hermann Böhlaus Nachfolger Weimar 2001

www. boehlausnf.de

info@boehlausnf.de 


\section{INHALTSVERZEICHNIS}

\section{HELMUT PFOTENHAUER}

Editorial.........................................................................

HELMUT PFOTENHAUER

Jean Paul - Ein Gegenklassiker. Eine Einführung .............. 4

JAN PHILLIP REEMTSMA

Komet

DURS GRÜNBEIN

Ein sarkastisches Kind

PETER SPRENGEL

Jean Pauls Antiklassizismus - Ein Rezeptionsphänomen? ...

HELMUT PFOTENHAUER

Das Leben schreiben - Das Schreiben leben. Jean Paul als Klassiker der Zeitverfallenheit

MONIKA SCHMITZ-EMANS

Der Komet als ästhetische Programmschrift - Poetologische Konzepte, Aporien und ein Sündenbock

HANS-WALTER SCHMIDT-HANNISA

„Der Traum ist unwillkürliche Dichtkunst“ - Traumtheorie und Traumaufzeichnung bei Jean Paul

THOMAS WIRTZ

Konstruiertes Leben. Bayle-Spuren im Werk Jean Pauls......

BARBARA HUNFELD

Glanz der Unebenheit. Aus Jean Pauls „Arbeitsloge“ des Hesperus. 
ELSBETH DANGEL-PELLOQUIN

Küsse und Risse: Jean Pauls Osculologie

BIRGIT SICK

Jean Pauls unveröffentlichte Satiren- und Ironienhefte (1782-1803)

BERNHARD BUSCHENDORF

„Um Ernst, nicht um Spiel wird gespielt.“ Zur relativen Autonomie des Ästhetischen bei Jean Paul

JOCHEN GOLZ

Blicke Jean Pauls auf Schiller

RALF SIMON

Versuch über einige Rahmenbedingungen des literarischen

Charakters in Jean Pauls Flegeljahren.

SABINE EICKENRODT

Horizontale Himmelfahrt. Die optische Metaphorik der Unsterblichkeit in Jean Pauls Komet

UWE JAPP

Die narrative Instanz des Humoristen in Dr. Katzenbergers Badereise

KURT WÖLFEL

Schoppe

Anschriften der Mitarbeiterinnen und Mitarbeiter 


\section{EDITORIAL}

Am 14. November 2000 war der 175. Todestag Jean Pauls zu begehen. Aus diesem Anlaß fand - neben zahlreichen anderen Veranstaltungen - vom 11. bis 14. Oktober in Bayreuth eine von der Jean-Paul-Gesellschaft ausgerichtete wissenschaftliche Tagung unter dem Motto „Jean Paul - ein Gegenklassiker" statt. Ihr ist diese Doppelnummer 2000/01 des Jahrbuchs der JeanPaul-Gesellschaft gewidmet.

Eingeleitet wurde die Tagung durch einen Festvortrag von Jan Philipp Reemtsma, begleitet wurde sie durch eine Lesung von Durs Grünbein, abgeschlossen mit einem Symphonie-Konzert der Tschechischen Philharmonie Brünn unter der Leitung von Caspar Richter, in dem Schumanns vierte und Mahlers erste Symphonie (mit dem später eliminierten zweiten Satz „Blumine") erklangen.

Zur Tagung wurde mit folgendem, als Anregung gedachten Text eingeladen:

Jean Paul - ein Gegenklassiker

Anläßlich des 175. Todestages von Jean Paul findet in Bayreuth vom 11.-14. Oktober 2000 eine wissenschaftliche Tagung unter dem Motto „Jean Paul ein Gegenklassiker"statt.

Die Themenformulierung geht davon aus, daß Jean Paul heute über die Zeiten hinweg eine merkwürdige Autorität zugebilligt wird, die sich gerade nicht aus den angeblichen Prädikaten des Klassischen wie Vollkommenheit, Rundung zum Ganzen, Mäßigung, Vermeidung der Extreme und dgl. herleitet. Hugo von Hofmannsthal bezeichnet Jean Paul als Klassiker der Formlosigkeit. Die Tagung geht davon aus, daß derlei Prädikationen in der eigentümlichen Unfertigkeit von Jean Pauls Schriften begründet ist, einer Virtualität, die zwar bemerkt, aber noch nicht ausreichend erklärt ist.

Zwei Aspekte könnten dazu dienen, die Frage danach zu präzisieren und zu differenzieren:

Die Formulierung „Gegenklassiker“ läßt zunächst denken an Jean Paul als Antiklassizisten. Die Opposition gegen die Schriftsteller, die man heute Klassiker nennt, hat ihn, so könnte man sagen, selbst zu einer Art Klassiker gemacht. Hier gilt es nun näher hinzuschauen: Die Gegenüberstellung von Kla ssizismus und Antiklassizismus um 1800 ist in der Forschung Differenzierungen bis hin zur Umkehr der Bedeutungen gewichen. Klassizismus trägt demnach das eigene Gegenteil selbst schon in sich: die Orientierung am Al- 
ten, eben Klassischen als ästhetischer Norm gebiert das Neue einer tendenziell selbstreferentiellen, vom Mimesisgebot und der Gegenstandshierarchie befreiten Kunst und Literatur. Und die Opposition dagegen bedient sich nicht nur bei den antiken Vorbildern, wie Jean Paul in der Titan-Zeit, sondern neigt ihrerseits zu jener Selbstbezüglichkeit des poetischen Verfahrens, die sie der Gegenseite vorwirft. Was bleibt also von jener Gegenüberstellung? Ist das Feldgeschrei nur eine Ablenkung vom anderen im eigenen, indem man es auf den Gegner projiziert und ihm lautstark diffamierend hinterherruft? Die Themenstellung wäre also nicht wörtlich zu nehmen, sondern Indikator für eine Lektüre gegen den Strich der Absichtserklärungen. In bezug auf Jean Paul hieße das zu fragen, inwiefern die Vorwürfe herz- und gegenstandsloser Formspielerei versteckte Selbstcharakteristiken wären, ästhetizistische Selbstermächtigungen durch die Verneinung hindurch. Die Figurationen, in denen sich diese Selbstgenügsamkeit des Ästhetischen auskristallisiert, sollen das faszinierende und gefährliche Potential autonomer Kunstübung unter Quarantäne stellen. Taugen sie dazu oder diffundiert das Inkriminierte in die Schreibweise, die es konstituiert und darüber hinaus? Es wäre also das Inventar des Klassizismusstreites noch einmal durchzumustern und auf die versteckten oder durch Polemik scheinbar entwerteten Kennzeichnungen von Jean Pauls literarischer Sprache abzuhorchen. Das Gegenstands- und Bodenlose könnte sich dann als triftig erweisen, ebenso wie das Ornamentale, das Orientalische, das Chinesische. Auch Jean Pauls Bollwerke gegen Ästhetizismus wie Empfindsamkeit, spirituelle Anthropologie, Unsterblichkeit wären dann vielleicht zu schleifen. Schließlich sind auch die scheinbar feststehenden Ausgrenzungen fraglich: Jean Paul und die Kunstgeschichte war bislang kein Thema, weil dies als die Domäne der Klassizisten galt; Jean Paul und Schiller galt als abgehakt, weil dieser, wie die Vorarbeiten zum Titan andeuten, in Don Gaspard eingekapselt schien.

Mit Klassizität ist die Vorstellung von Zeitenthobenheit verbunden. Zu prüfen wäre, ob Jean Pauls gegenläufige Geltung nicht in einer Art Klassizität der Zeitverfallenheit bestehen könnte. Jean Pauls Schriften sind in geradezu obsessiver Weise dem Ablaufen der Zeit und dem Tod gewidmet. Vielleicht besteht in diesem unaufhörlichen Anschreiben gegen die Kränkung des Zufalls von Zeugung und Geburt, von Körperlichkeit, Altern und Endlichkeit, im Aussichtslosen also im Grunde, seine Zeitresistenz und der dauernde Impuls seines Schreibens - mehr vielleicht als in den großen, ins Affirmative gewendeten Botschaften, wie Unsterblichkeit, Seele, Unendlichkeit? - Nicht nur thematische Bereiche wie Erinnern, Vergessen, Zeugung, Selbstzeugung, Geburt und ihre litterale Komplementierung durch Autorschaft, Tod und Weiterleben als Schrift, wären da einschlägig. Vielmehr wäre auch Jean Pauls eigentümliche Abneigung oder Unfähigkeit, Geschichten zu Ende zu erzählen, auf die Priorität des dauernden Schreibens gegenüber der Vergegenständlichung in der abgerundeten Handlung oder im geschlossenen Werk hin zu durchdenken. Auch jene Selbstreferentialität, die ständige Selbstthematisierung des Schreibens und des Schreibers, könnten als Immunisierung gegen 
die Zeit gedeutet werden. Eine Buchstabenwelt mit ihren inneren Bezüglichkeiten würde demnach gegen die heteronome Welt und ihren linearen, unerbittlichen Verlauf gestellt.

Von den vorgesehenen 17 Referenten mußten bedauerlicherweise Günter Oesterle und Norbert Miller kurzfristig absagen. Mehrere interessante Themenvorschläge, die später eingereicht wurden, konnten leider nicht mehr berücksichtigt werden. Die Jean-Paul-Forschung ist - erfreulicherweise - umfänglicher und aspektreicher als solch eine Tagung es dokumentieren kann. Die folgenden Jahrbücher werden dem hoffentlich Rechnung tragen können.

Der Veranstalter verdankt Thomas Wirtz von der FAZ Unterstützung bei der Vorbereitung und der Leitung der Tagung. Zu danken ist dem Vizepräsidenten der Gesellschaft, Herrn Friedrich, der in Bayreuth alles Erdenkliche tat, um zum Gelingen der Tagung beizutragen. Mein Dank gilt schließlich und ganz besonders Frau Hunfeld, der Mitarbeiterin an der Würzburger JeanPaul-Arbeitsstelle, die die Hauptlast der Vorbereitung trug.

Eine Veranstaltung wie die hier dokumentierte ist nicht möglich ohne Sponsoren. Sie seien hier abschließend dankbar genannt. Es sind dies:

die Stadt Bayreuth,

die Fritz-Thyssen-Stiftung,

die Arbeitsgemeinschaft literarischer Gesellschaften, der Bezirk Oberfranken, die Oberfrankenstiftung, die Kreissparkasse Bayreuth-Pegnitz sowie der Rotary-Club Bayreuth-Eremitage.

Würzburg, im November 2000 